
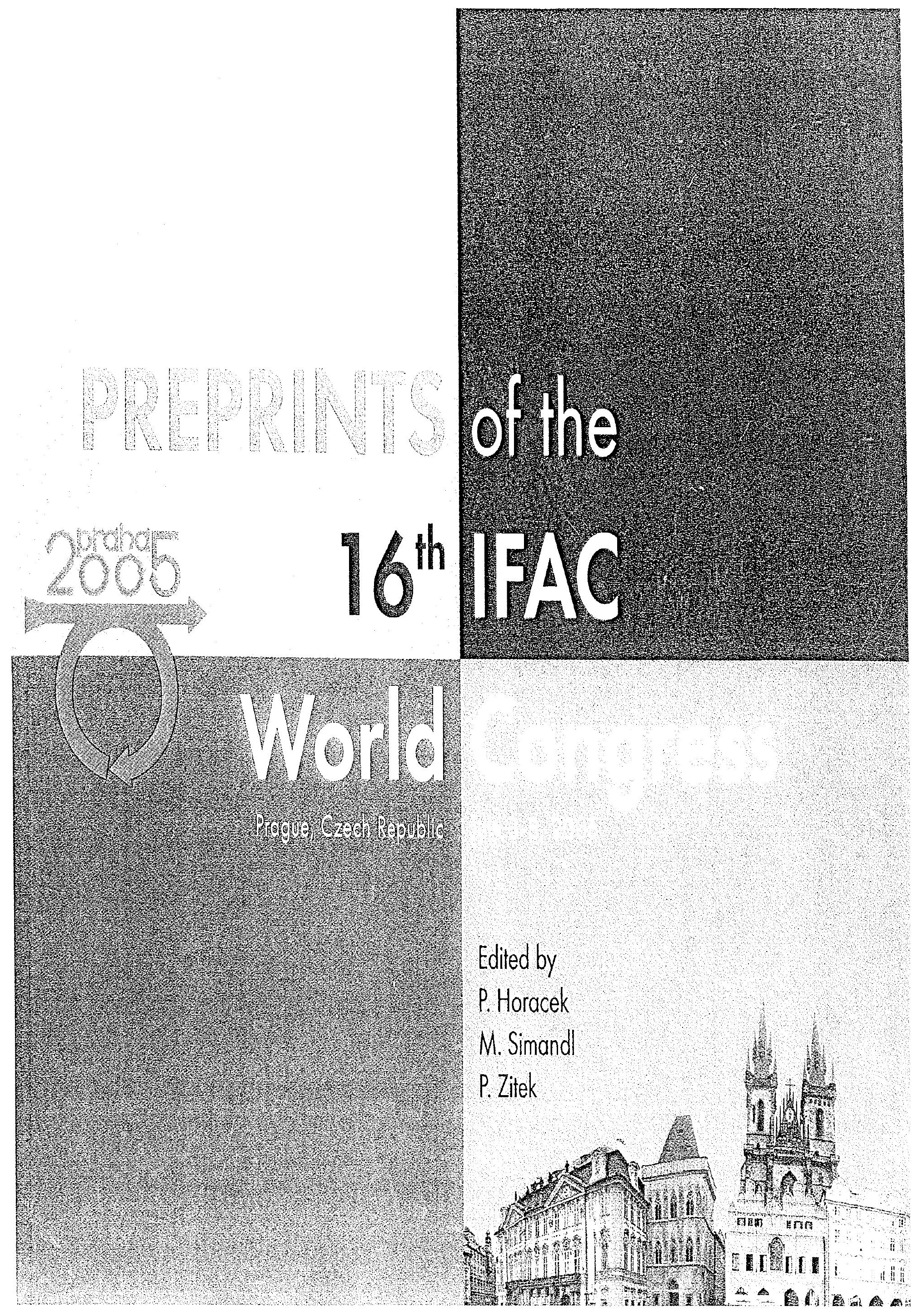


\title{
A NEW CONCEPT OF INVARIANCE FOR SATURATED SYSTEMS
}

\author{
T. Alamo ${ }^{\dagger} *$ A. Cepeda * D. Limon * E.F. Camacho* \\ * Departamento de Ingeniería de Sistemas y Automática, \\ Universidad de Sevilla. \\ Escuela Superior de Ingenieros, Camino de los Descubrimientos \\ s/n. 41092 Sevilla, SPAIN. \\ Telephone: +34 954487347, Fax: +34954487340 \\ \{alamo,cepeda,limon,eduardo\}@cartuja.us.es
}

\begin{abstract}
In this paper, a new concept of invariance for saturated linear systems is presented. This new notion of invariance, denoted SNS-invariance, has a number of geometrical properties that makes its use suitable for the estimation of the domain of attraction of saturated systems. The notion of SNS-domain of attraction, that serves as an estimation of the domain of attraction of a saturated system, is introduced. It is shown that, in case of single input saturated systems, any contractive set is contained in the SNSdomain of attraction. A simple algorithm that converges to the SNS-domain of attraction is presented. Some illustrative examples are given.
\end{abstract}

Copyright(C)2005 IFAC

Keywords: Saturated System, Set Invariance, Domain of Attraction

\section{INTRODUCTION}

Saturation is probably the most commonly encountered nonlinearity in control engineering. For this reason, the estimation of the domain of attraction of linear systems subject to control saturation have received the attention of many authors in the last years (see, for example, (Gomes Da Silva Jr. and Tarbouriech, 1999; Milani, 2002; Hu and Lin, 2001) and references therein).

The domain of attraction of a saturated system can be estimated by means of a linear difference inclusion (LDI) of the system. The politopic representation provided by the LDI simplifies the analysis of the non-linear system. In (Gomes Da Silva Jr. and Tarbouriech, 2001), an LDI is used to obtain invariant ellipsoids for saturated systems.

\footnotetext{
$\star \dagger$ Corresponding author
}

The linear difference inclusion presented in (Gomes Da Silva Jr. and Tarbouriech, 2001) is generalized in ( $\mathrm{Hu}$ and Lin, 2001) and ( $\mathrm{Hu}$ et al., 2002). Based on this generalization, the authors propose how to choose an auxiliary matrix, that characterizes the LDI, in order to obtain the greatest ellipsoid that is invariant under the corresponding LDI. This LDI representation has also been used in (Cao and Lin, 2003) to obtain a saturation-dependent Lyapunov function that leads to a less conservative estimation of the domain of attraction.

In this paper a new notion of invariance, denoted as SNS-invariance, is presented. Based on its geometrical properties, a simple algorithm to estimate the domain of attraction of a saturated linear system is proposed. It is shown that in case of single input systems, any contractive set is a SNS-invariant set.

The paper is organized as follows. In section 2 the class of saturated systems considered is presented. The 
new notion of invariance is introduced in section 3 . A polyhedral representation of the SNS-one-step set operator is given in section 4. Based on this operator, an algorithm that converges to the SNS-domain of attraction is proposed in section 5. Some illustrative examples are given in section 6 . The paper draws to a close with a section of conclusions.

\section{PROBLEM STATEMENT}

Consider the following system

$$
x^{+}=A x+B \sigma(K x)
$$

where $x \in \mathbb{R}^{n}$ denotes the state vector. The function $\sigma: \mathbb{R}^{m} \rightarrow \mathbb{R}^{m}$ is the vector-valued standard saturation function defined as follows:

$$
\sigma(u)=\left[\begin{array}{llll}
\sigma\left(u_{1}\right) & \sigma\left(u_{2}\right) & \ldots & \sigma\left(u_{m}\right)
\end{array}\right]^{\top},
$$

where $\sigma\left(u_{i}\right)=\operatorname{sign}\left(u_{i}\right) \min \left\{1,\left|u_{i}\right|\right\}$.

Denote $\mathcal{M}=\{1,2, \ldots, m\}$. Denote also $B_{i}, i=1, \ldots, m$ the columns of matrix $B$ and $K_{i}, i=1, \ldots, m$ the rows of matrix $K$. With this notation, system (1) can be rewritten as:

$$
x^{+}=A x+\sum_{i=1}^{m} B_{i} \sigma\left(K_{i} x\right)=A x+\sum_{i \in \mathcal{M}} B_{i} \sigma\left(K_{i} x\right)
$$

\section{DEFINING THE NOTION OF SNS-INVARIANCE}

In this section, the concept of SNS-invariance is presented. For this purpose, some auxiliary notation is required:

Definition 1. Given a set of integers $T$, set $\mathcal{V}_{T}$ is the set of all subsets of $T$. That is,

$$
\mathcal{V}_{T}=\{S: S \subseteq T\}
$$

Example: If $m=2$, then $\mathcal{M}=\{1,2\}$ and $\mathcal{V}_{\mathcal{M}}=$ $\{\emptyset,\{1\},\{2\},\{1,2\}\}$. Note that the empty set $\emptyset$ belongs to $\mathcal{V}_{\mathcal{M}}$.

Throughout this paper: $S^{c}$ denotes the complementary of $S$ in $\mathcal{M}$. That is, $S^{c}=\{i \in \mathcal{M}: i \notin S\}$.

Definition 2. Given a set $S \in \mathcal{V}_{\mathcal{M}}, F(x, S)$ is defined as follows:

$$
F(x, S)=A x+\sum_{i \in S^{c}} B_{i} K_{i} x+\sum_{i \in S} B_{i} \sigma\left(K_{i} x\right)
$$

Note that with these definitions, $x^{+}=A x+B \sigma(K x)=$ $F(x, \mathcal{M})$. Also, $x^{+}=F(x, \emptyset)=(A+B K) x$ represents the evolution of the system without saturation.

The notion of SNS-invariance is introduced in the following definition:
Definition 3. A set $\Omega$ is said to be SNS-invariant for system $x^{+}=A x+B \sigma(K x)$ if $x \in \Omega$ implies $F(x, S) \in \Omega$ for every $S \in \mathcal{V}_{\mathcal{M}}$.

It is clear from the previous definition that if $\Omega$ is a SNS-invariant set then $\Omega$ is an invariant set for the saturated system $x^{+}=A x+B \sigma(K x)$. In effect, if $\Omega$ is SNS-invariant then $A x+B \sigma(K x)=F(x, \mathcal{M}) \in \Omega$, for all $x \in \Omega$.

For single input systems $(m=1)$, the SNS-invariance of a given set $\Omega$ is equivalent to the invariance of $\Omega$ for the Saturated and Non Saturated systems: $x^{+}=A x+$ $B \sigma(K x)$ and $x^{+}=A x+B K x$. Note that $S N S$ stands for Saturated and Non Saturated.

\subsection{Geometric condition of SNS-invariance}

In the context of set invariance theory, the one-step set plays an important role (Blanchini, 1999). This concept, for the saturated system (1), is introduced in the following definition.

Definition 4. Given system $x^{+}=A x+B \sigma(K x)$ and set $\Omega$, the one-step set $Q(\Omega)$ is defined as

$$
Q(\Omega)=\{x: A x+B \sigma(K x) \in \Omega\}
$$

It is well known that $\Omega$ is an invariant set for system $x^{+}=A x+B \sigma(K x)$ if and only if $\Omega \subseteq Q(\Omega)$ (Blanchini, 1999). Given a convex set $\Omega$, the one-step set $Q(\Omega)$ is not necessarily convex due to the nonlinear nature of the saturation function. The non convex nature of $Q(\Omega)$ makes it difficult to use operator $Q(\cdot)$ in the computation of invariant sets for saturated systems.

In order to provide a geometric condition of SNSinvariance, the following definitions are introduced.

Definition 5. Given a set $\Omega$ and $S \in \mathcal{V}_{\mathcal{M}}$ :

$$
Q_{S}(\Omega)=\{x: F(x, S) \in \Omega\},
$$

and given a set $\Omega$ :

$$
Q_{S N S}(\Omega)=\bigcap_{S \in \mathcal{V}_{\mathcal{M}}} Q_{S}(\Omega)
$$

From the definition of $Q_{S N S}(\cdot)$, the following property is directly inferred:

Property 1. A set $\Omega$ is SNS-invariant if and only if $\Omega \subseteq Q_{S N S}(\Omega)$.

The most remarkable property of $Q_{S N S}(\cdot)$ is that given a polyhedral set $\Omega, Q_{S N S}(\Omega)$ is a convex polyhedron. This striking property will be proved in the following section. 


\section{POLYHEDRAL REPRESENTATION OF $Q_{S N S}(\Omega)$}

In this section, a polyhedral representation of $Q_{S N S}(\Omega)$ is provided. Given a polyhedral set $\Omega$ and a set $S$ in $\mathcal{V}_{\mathcal{M}}, Q_{S}(\Omega)$ is not necessarily a polyhedral set. Surprisingly,

$$
Q_{S N S}(\Omega)=\bigcap_{S \in \mathcal{V}_{\mathcal{M}}} Q_{S}(\Omega)
$$

is a polyhedral set that can be obtained in a direct way from polyhedron $\Omega$ as it is claimed in the following theorem.

Theorem 1. Let us suppose that $\Omega$ is a convex polyhedron in $\mathbb{R}^{n}$ given by $\Omega=\{x: R x \preceq g\}$. Then:

$Q_{S N S}(\Omega)=\bigcap_{S \in \mathcal{V}_{\mathcal{M}}}\left\{x: R\left(A+\sum_{i \in S^{c}} B_{i} K_{i}\right) x-\sum_{i \in S}\left|R B_{i}\right| \preceq g\right\}$

where $S^{c}$ denotes the complementary of $S$ in $\mathcal{M}$ and $\left|R B_{i}\right|$ is the vector with entries equal to the absolute value of the entries of vector $R B_{i}$.

ProOF: See appendix A.

\section{SNS-DOMAIN OF ATTRACTION}

In this section, the notion of SNS-domain of attraction is introduced. It is shown that the SNS-domain of attraction is included in the domain of attraction of the saturated system. Taking into account the results of the previous section, a simple algorithm that converges to the SNS-domain of attraction of the system is proposed. It is also shown in this section that, in case of single input systems, any contractive set belongs to the SNS-domain of attraction.

Definition 6. A sequence $\left\{S_{0}, S_{1}, S_{2}, \ldots\right\}$ is admissible if all the elements of the sequence belong to $\mathcal{V}_{\mathcal{M}}$.

Definition 7. The initial condition $x_{0}$ belongs to the SNS-domain of attraction of system $x^{+}=A x+$ $B \sigma(K x)$ if the recursion

$$
x_{k+1}=F\left(x_{k}, S_{k}\right)
$$

converges to the origin for any admissible sequence $\left\{S_{0}, S_{1}, S_{2}, \ldots\right\}=\left\{S_{k}\right\}_{0}^{\infty}$.

It is clear from the previous definition that the SNSdomain of attraction is included in the domain of attraction of the saturated system. The following theorem states that it is possible to obtain the SNS-domain of attraction by means of a simple recursion.

Theorem 2. Denote $\mathcal{L}(K)$ the region of linear behaviour of the saturated system, that is, $\mathcal{L}(K)=\{x \in$ $\left.\mathbb{R}^{n}:\|K x\|_{\infty} \leq 1\right\}$. Suppose that $\Phi \subseteq \mathcal{L}(K)$ is a convex polyhedron with non zero volume. Suppose also that $\Phi$ is an invariant set for the asymptotically stable system $x^{+}=(A+B K) x$. Denote now $C_{0}=\Phi$ and consider the following recursion:

$$
C_{k+1}=Q_{S N S}\left(C_{k}\right)
$$

Then:

(i) $C_{k}$ is a convex polyhedron that can be obtained by means of theorem $1, \forall k \geq 1$.

(ii) $C_{k}$ is a SNS-invariant set for system $x^{+}=A x+$ $B \sigma(K x), \forall k \geq 0$.

(iii) $C_{k} \subseteq C_{k+1}, \forall k \geq 0$.

(iv) $C_{k}$ belongs to the SNS-domain of attraction of system $x^{+}=A x+B \sigma(K x), \forall k \geq 0$.

(v) The sequence $\left\{C_{0}, C_{1}, C_{2}, \ldots\right\}$ converges to the SNS-domain of attraction of system $x^{+}=A x+$ $B \sigma(K x)$.

(vi) The SNS-domain of attraction of the saturated system $x^{+}=A x+B \sigma(K x)$ is a convex set.

\section{ProOF :}

(i) Theorem 1 states that if $\Omega$ is a convex polyhedron then $Q_{S N S}(\Omega)$ is also a convex polyhedron. This, and the fact that $C_{0}$ is a convex polyhedron, prove that the recursion $C_{k+1}=Q_{S N S}\left(C_{k}\right)$ always yields convex polyhedrons.

(ii) As $C_{0}$ belongs to $\mathcal{L}(K)$ it results that $F(x, S)=$ $(A+B K) x$, for all $x \in C_{0}$ and for all $S \in \mathcal{V}_{\mathcal{M}}$. From this and the invariance of $C_{0}$ it is inferred that $F(x, S) \in C_{0}$ for all $x \in C_{0}$ and for all $S \in$ $\mathcal{V}_{\mathcal{M}}$; that is to say, $C_{0}$ is SNS-invariant.

Let us now suppose that $C_{k-1}$ is SNS-invariant, then $C_{k-1} \subseteq Q_{S N S}\left(C_{k-1}\right)=C_{k}$ (see property 1 ). Therefore, if $x \in C_{k}=Q_{S N S}\left(C_{k-1}\right)$ then $F(x, S) \in$ $C_{k-1} \subseteq C_{k}$, for all $S \in \mathcal{V}_{\mathcal{M}}$.

(iii) From the geometric condition of SNS-invariance (see property 1): $C_{k} \subseteq Q_{S N S}\left(C_{k}\right)=C_{k+1}$

(iv) From the SNS-invariance of $C_{0} \subseteq \mathcal{L}(K)$ and the asymptotically stability of the non saturated system it is inferred that $C_{0}$ belongs to the SNSdomain of attraction of the system. Note that if $C_{k-1}$ belongs to the SNS-domain of attraction then $C_{k}=Q_{S N S}\left(C_{k-1}\right)$ also belongs to the SNSdomain of attraction. This is due to the fact that $F(x, S) \in C_{k-1}$, for all $x \in C_{k}$ and for all $S \in \mathcal{V}_{\mathcal{M}}$. Therefore, the recursion $C_{k+1}=Q_{S N S}\left(C_{k}\right)$ with $C_{0}=\Phi$ yields SNS-invariant sets that belong to the SNS-domain of attraction.

(v) Suppose now that $x$ belongs to the SNS-domain of attraction of the system. As $\Phi$ is an invariant set with nonzero volume, there exists $p$ such that the recursion $x_{k+1}=F\left(x_{k}, S_{k}\right)$ with $x_{0}=x$ satisfies $x_{p} \in \Phi=C_{0}$ for all admissible sequence $S_{0}, S_{1}, \ldots, S_{p}$. This is equivalent to say that $x$ is included in $C_{p}$ and, consequently, $x$ belongs to the SNS-domain of attraction. 
(vi) It suffices to show that given two points $x_{1}$ and $x_{2}$ belonging to the SNS-domain of attraction, $\lambda x_{1}+(1-\lambda) x_{2}$ belongs to the SNS-domain of attraction for every $\lambda \in[0,1]$. If $x_{1}$ and $x_{2}$ belong to the SNS-domain of attraction then it is clear from the previous claim that there exists $p_{1}$ and $p_{2}$ such that $x_{1} \in C_{p_{1}}, x_{2} \in C_{p_{2}}$. Denote now $p=$ $\max \left\{p_{1}, p_{2}\right\}$, taking into account that $C_{k} \subseteq C_{k+1}$, $\forall k \geq 0$, it is inferred that $x_{1} \in C_{p}$ and $x_{2} \in C_{p}$. From the fact that $C_{p}$ is a convex set contained in the SNS-domain of attraction of the system it is concluded that $\lambda x_{1}+(1-\lambda) x_{2}$ belongs to $C_{p}$ and therefore to the SNS-domain of attraction for every $\lambda \in[0,1]$.

The recursion presented in theorem 2 requires an invariant set of the linear system $x^{+}=A x+B K x$, included in $\mathcal{L}(K)$. Note that this admissible invariant set can be obtained by standard algorithms (see (Gilbert and Tan, 1991; Blanchini, 1999)).

Let us consider any set $C_{k}$ obtained from the recursion presented in theorem 2; any set included in $C_{k}$ belongs to the SNS-domain of attraction. For example, an ellipsoidal inner approximation of set $C_{k}$ serves as an estimation of the domain of attraction of the saturated system. From the convexity of the SNS-domain of attraction it is inferred that the convex hull of a given collection of sets belonging to the SNS-domain of attraction also belongs to the SNS-domain of attraction.

The following property states, for single input systems, that any contractive set of the saturated system belongs to the SNS-domain of attraction. This means that the maximal contractive set for a given single input system is characterized, in a non conservative way, by the recursion proposed in theorem 2 .

Property 2. Let us suppose that $m=1$ (single input case), and that $\Omega$ is a contractive set for system (1). That is, there is $\lambda \in[0,1)$ such that $x \in \varepsilon \Omega$ implies $A x+B \sigma(K x) \in \lambda \varepsilon \Omega, \forall \varepsilon \in[0,1]$. Then $\Omega$ is a SNSinvariant set that belongs to the SNS-domain of attraction of the system.

\section{PROOF :}

It will be first shown that if $x \in \varepsilon \Omega$ then $F(x, S) \in \lambda \varepsilon \Omega$ for all $S \in \mathcal{V}_{\mathcal{M}}=\{\emptyset, 1\}$. That is, $A x+B \sigma(K x) \in \lambda \varepsilon \Omega$ and $A x+B K x \in \lambda \varepsilon \Omega$. The first inclusion is clear from the assumptions of the property. It is now shown that $A x+B K x \in \lambda \varepsilon \Omega$. In effect, if $x \in \varepsilon \Omega$ then there exists $\gamma \in(0,1]$ such that $|K \gamma x| \leq 1$. Moreover, as $x \in \varepsilon \Omega$, it results that $\gamma x \in \gamma \varepsilon \Omega$. From the assumptions of the property, it can be now concluded that :

$$
A \gamma x+B K \gamma x=A \gamma x+B \sigma(K \gamma x) \in \lambda \gamma \varepsilon \Omega
$$

Note that $A \gamma x+B K \gamma x \in \lambda \gamma \varepsilon \Omega$ implies $A x+B K x \in$ $\lambda \varepsilon \Omega$. It has then been proved that $x \in \varepsilon \Omega, \varepsilon \in[0,1]$ implies:

$$
F(x, S) \in \lambda \varepsilon \Omega, \quad \forall S \in \mathcal{V}_{\mathcal{M}}=\{\emptyset, 1\} .
$$

It is clear that this implies that $\Omega$ is SNS-invariant.

In what follows it is shown that $\Omega$ belongs to the

recursion: $x_{k+1}=F\left(x_{k}, S_{k}\right)$ with $x_{0}=x \in \Omega$. From the previous discussion it is clear that $x_{k} \in \lambda^{k} \Omega$, for every admissible sequence $S_{0}, S_{1}, \ldots, S_{k-1}$. Therefore, $\lim _{k \rightarrow \infty} x_{k}=0$, for every admissible sequence $\left\{S_{k}\right\}_{0}^{\infty}$. This proves the claim.

\section{NUMERICAL EXAMPLES}

\subsection{Example 1}

Let us consider the system $x^{+}=A x+B \sigma(K x)$ with

$$
A=\left[\begin{array}{ll}
1 & 1 \\
0 & 1
\end{array}\right], B=\left[\begin{array}{c}
0.5 \\
1
\end{array}\right], K=[-0.6167-1.2703]
$$

Figure (1) shows the sequence $\left\{C_{0}, C_{1}, \ldots, C_{27}\right\}$ provided by the recursion of theorem (2): $C_{k+1}=Q_{S N S}\left(C_{k}\right)$. The sequence starts with an invariant set $\Phi=C_{0}$ contained in the region of linear behaviour of the system (shadowed in the figure). The sequence leads to the SNS-domain of attraction of the system. The SNSinvariant sets $C_{k}, k=1, \ldots, 27$, are displayed in the figure (note that, as it is claimed in theorem $2, C_{k} \subseteq C_{k+1}$, $\forall k \geq 0$ ).

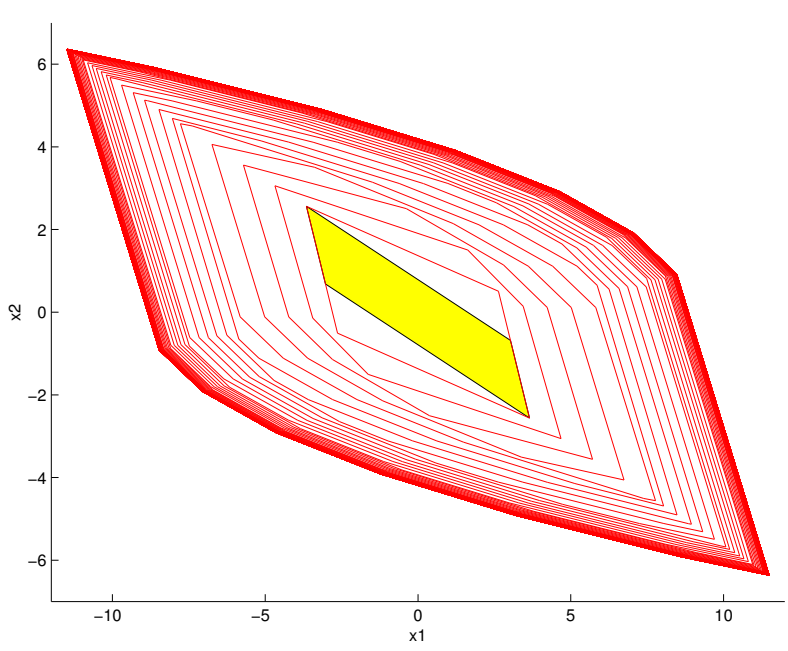

Fig. 1. Sequence $C_{k}$ leading to the SNS-domain of attraction.

The domain of attraction of this system has been estimated by means of a saturation-dependent Lyapunov function in (Cao and Lin, 2003). In that paper, the authors propose how to choose matrix $H$ (that characterizes a linear difference inclusion of the saturation nonlinearity) in such a way that a saturation-dependent 
Lyapunov function is strictly decreasing. Therefore, the authors are obtaining an estimation of the domain of attraction of the system by means of the concept of linear difference inclusion.

Figure (2) compares the SNS-domain of attraction with the estimation obtained by means of a saturationdependent Lyapunov function (Cao and Lin, 2003). This figure shows that the SNS-domain of attraction contains the estimation provided by the LDI approach.

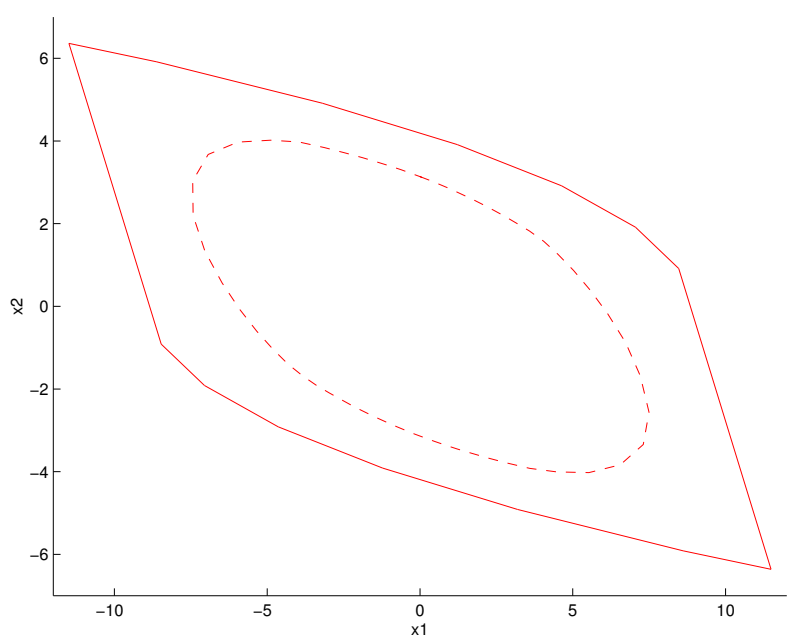

Fig. 2. SNS-domain of attraction (solid line) and estimation of the domain of attraction obtained by means of a saturation-dependent Lyapunov function (dotted line).

\subsection{Example 2}

Consider the system $x^{+}=A x+B \sigma(K x)$, with:

$A=\left[\begin{array}{cc}1.2 & 0 \\ 0.4 & 0.5\end{array}\right], \quad B=\left[\begin{array}{ll}2 & 0 \\ 0 & 2\end{array}\right], \quad K=\left[\begin{array}{cc}-0.475 & 0 \\ 0.55 & 0.075\end{array}\right]$

This example was introduced in (Gomes Da Silva Jr. and Tarbouriech, 1999). In that paper, the authors use an initial contractive polyhedral set $\Upsilon \subseteq \mathcal{L}(K)$ and show how to enlarge it in such a way that the contractiveness of the enlarged polyhedron is maintained. That is, the maximum value of the scalar $\alpha$ such that $\alpha \Upsilon$ is a contractive polyhedron for the saturated system is obtained. Using this approach, the authors showed that the region $\left\{x:\|x\|_{\infty} \leq 10\right\}$ is included in the domain of attraction of the system.

Figure (3) shows the sequence $\left\{C_{0}, C_{1}, \ldots, C_{25}\right\}$ provided by the recursion of theorem (2). The domain of attraction of the system of this example is $\Gamma=$ $\left\{x \in \mathbb{R}^{2}:\left|x_{1}\right|<10\right\}$. It can be shown that, in this particular case, the sequence $\left\{C_{k}\right\}$ converges not only to the SNS-domain of attraction of the system but also to the actual domain of attraction.

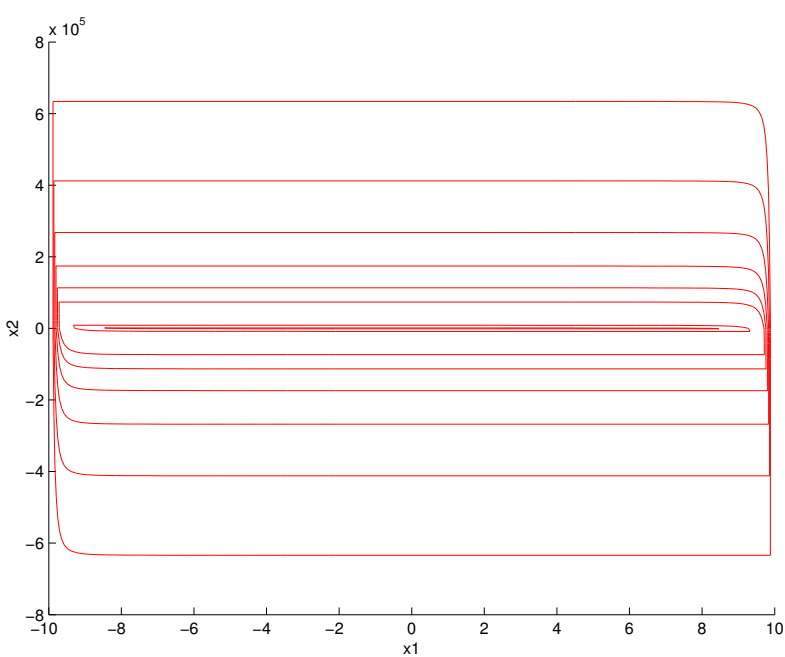

Fig. 3. Sequence $C_{k}$ leading to the SNS-domain of attraction of the system.

\section{CONCLUSIONS}

In this paper a new notion of SNS-invariance is introduced. The geometrical properties of the SNSinvariance concept leads to the definition of the SNSdomain of attraction of a given saturated system. A recursive algorithm that converges to the SNS-domain of attraction is presented. One of the most remarkable properties of the SNS-domain of attraction is that any contractive set for a saturated single input system is included in the SNS-domain of attraction. Numerical examples demonstrate the effectiveness of the new approach.

\section{Appendix A. PROOF OF THEOREM 1}

Denote

$$
P_{S N S}=\bigcap_{S \in \mathcal{V}_{\mathcal{M}}}\left\{x: R\left(A+\sum_{i \in S^{c}} B_{i} K_{i}\right) x-\sum_{i \in S}\left|R B_{i}\right| \preceq g\right\}
$$

In what follows, it will be proven that $P_{S N S} \subseteq Q_{S N S}(\Omega)$. Let us suppose that there is $\hat{x} \in P_{S N S}$ such that $\hat{x} \notin$ $Q_{S N S}(\Omega)$. That is, there is $\hat{S} \in \mathcal{V}_{\mathcal{M}}$ such that $\hat{x} \notin Q_{\hat{S}}(\Omega)$. In this case, there must be $j$ such that denoting $R_{j}$ and $g_{j}$ the $j$-esime row of $R$ and $j$-esime component of $g$ respectively:

$$
\begin{aligned}
& R_{j} F(\hat{x}, \hat{S})= \\
& R_{j}\left(\left(A+\sum_{i \in \hat{S}^{c}} B_{i} K_{i}\right) \hat{x}+\sum_{i \in \hat{S}} B_{i} \sigma\left(K_{i} \hat{x}\right)\right)>g_{j}
\end{aligned}
$$

It will be shown that the above inequality contradicts the fact that $\hat{x} \in P_{S N S}$. In effect, taking into account that $a \sigma(y) \leq \max \{a y,-|a|\}$ (see appendix B ):

$$
\begin{aligned}
& R_{j} F(\hat{x}, \hat{S})=R_{j}\left(A+\sum_{i \in \hat{S}^{c}} B_{i} K_{i}\right) \hat{x}+\sum_{i \in \hat{S}} R_{j} B_{i} \sigma\left(K_{i} \hat{x}\right) \\
\leq & R_{j}\left(A+\sum_{i \in \hat{S}^{c}} B_{i} K_{i}\right) \hat{x}+\sum_{i \in \hat{S}} \max \left\{R_{j} B_{i} K_{i} \hat{x},-\left|R_{j} B_{i}\right|\right\}
\end{aligned}
$$


Denote:

$$
T=\left\{i \in \hat{S}: R_{j} B_{i} K_{i} \hat{x}<-\left|R_{j} B_{i}\right|\right\}
$$

From this definition and the previous inequality:

$$
\begin{aligned}
& R_{j} F(\hat{x}, \hat{S}) \leq R_{j}\left(A+\sum_{i \in \hat{S}^{c}} B_{i} K_{i}\right) \hat{x} \\
& +\sum_{i \in \hat{S}, i \notin T} R_{j} B_{i} K_{i} \hat{x}-\sum_{i \in T}\left|R_{j} B_{i}\right| \\
& =R_{j}\left(A+\sum_{i \in \mathcal{M}, i \notin \hat{S}} B_{i} K_{i}+\sum_{i \in \hat{S}, i \notin T} B_{i} K_{i}\right) \hat{x}-\sum_{i \in T}\left|R_{j} B_{i}\right| \\
& =R_{j}\left(A+\sum_{i \in \mathcal{M}} B_{i} K_{i}-\sum_{i \in \hat{S}} B_{i} K_{i}+\sum_{i \in \hat{S}} B_{i} K_{i}-\sum_{i \in T} B_{i} K_{i}\right) \hat{x} \\
& -\sum_{i \in T}\left|R_{j} B_{i}\right| \\
& =R_{j}\left(A+\sum_{i \in \mathcal{M}} B_{i} K_{i}-\sum_{i \in T} B_{i} K_{i}\right) \hat{x}-\sum_{i \in T}\left|R_{j} B_{i}\right| \\
& =R_{j}\left(A+\sum_{i \in T^{c}} B_{i} K_{i}\right) \hat{x}-\sum_{i \in T}\left|R_{j} B_{i}\right|
\end{aligned}
$$

It is clear that $T \in \mathcal{V}_{\mathcal{M}}$. Thus $\hat{x} \in P_{S N S}$ implies:

$$
R\left(A+\sum_{i \in T^{c}} B_{i} K_{i}\right) \hat{x}-\sum_{i \in T}\left|R B_{i}\right| \preceq g
$$

In particular,

$$
R_{j}\left(A+\sum_{i \in T^{c}} B_{i} K_{i}\right) \hat{x}-\sum_{i \in T}\left|R_{j} B_{i}\right| \leq g_{j}
$$

This inequality contradicts equation (A.1). Therefore, it is inferred that $R_{j} F(\hat{x}, \hat{S}) \leq g_{j}$ and consequently: $P_{S N S} \subseteq Q_{S N S}(\Omega)$.

To conclude the prove, it will be shown that $Q_{S N S}(\Omega) \subseteq$ $P_{S N S}$. In effect, due to the fact that $-\left|R B_{i}\right| \preceq R B_{i} \sigma\left(K_{i} x\right)$, it results that, for every $S \in \mathcal{V}_{\mathcal{M}}$ :

$$
\begin{gathered}
R\left(A+\sum_{i \in S^{c}} B_{i} K_{i}\right) x-\sum_{i \in S}\left|R B_{i}\right| \preceq \\
R\left(A+\sum_{i \in S^{c}} B_{i} K_{i}\right) x+\sum_{i \in S} R B_{i} \sigma\left(K_{i} x\right)=R F(x, S)(\mathrm{A} .2)
\end{gathered}
$$

Suppose now that $x \in Q_{S N S}(\Omega)$, that is, $R F(x, S) \preceq g$, $\forall S \in \mathcal{V}_{\mathcal{M}}$. Then, taking into account equation (A.2), it results that

$R\left(A+\sum_{i \in S^{c}} B_{i} K_{i}\right) x-\sum_{i \in S}\left|R B_{i}\right| \preceq R F(x, S) \preceq g, \forall S \in \mathcal{V}_{\mathcal{M}}$

It is concluded that $x \in Q_{S N S}(\Omega)$ implies $x \in P_{S N S}$. This proves the claim.
Appendix B.

Property 3. Given $a \in \mathbb{R}$ and $y \in \mathbb{R}$ :

$$
a \sigma(y) \leq \max \{a y,-|a|\}
$$

PROOF :

1. $|y| \leq 1: \max \{a y,-|a|\}=a y=a \sigma(y)$

2. $|y|>1$ and $a y \geq 0: \max \{a y,-|a|\}=a y \geq$ $a \operatorname{sign}(y)=a \sigma(y)$

3. $|y|>1$ and $a y<0: \max \{a y,-|a|\}=-|a|=$ $a \operatorname{sign}(-a)=a \operatorname{sign}(y)=a \sigma(y)$

\section{REFERENCES}

Blanchini, F. (1999). Set invariance in control. Automatica 35, 1747-1767.

Cao, Yong-Yan and Zongli Lin (2003). Stability analysis of discrete-time systems with actuator saturation by a saturation-dependent lyapunov function. Automatica 39, 1235-1241.

Gilbert, E.G. and K.T. Tan (1991). Linear systems with state and control constraints: The theory and application of maximal output admissible sets. IEEE Transactions on Automatic Control 36, 1008-1020.

Gomes Da Silva Jr., J. M. and S. Tarbouriech (1999). Polyhedral regions of local asymptotic stability for discrete-time linear systems with saturating controls. IEEE Transactions on Automatic Control 44(11), 2081-2085.

Gomes Da Silva Jr., J. M. and S. Tarbouriech (2001). Local stabilization of discrete-time linear systems with saturatings controls: an LMI-based approach. IEEE Transactions on Automatic Control 46, 119-125.

Hu, T. and Z. Lin (2001). Control Systems with Actuator Saturation. Analysis and Design. Birkhäuser.

$\mathrm{Hu}$, Tingshu, Zongli Lin and Ben M. Chen (2002). Analysis and design for discrete-time linear systems subject to actuator saturation. Systems \& Control Letters 45, 97-112.

Milani, Basilio E.A. (2002). Piecewise-affine lyapunov functions for discrete-time linear systems with saturating controls. Automatica 38, 21772184. 